\title{
POROUS SETS AND NULL SETS FOR ELLIPTIC HARMONIC MEASURES
}

\author{
JANG-MEI WU
}

\begin{abstract}
We give a genuinely $n$-dimensional construction of uniformly elliptic operators $L$ in $\mathbb{R}_{+}^{n}$ (of divergence form, and of nondivergence form), which have positive $L$-harmonic measures on a class of porous sets on $\partial \mathbb{R}_{+}^{n}$ with zero surface measure. The porosity condition given is sharp. The earlier methods were all two dimensional.
\end{abstract}

\section{INTRODUCTION}

In a sequence of papers ([1], [6], [11], and [12]) Modica, Mortola, Salsa, Caffarelli, Fabes, Kenig, and Barceló have constructed elliptic operators of the forms $(0.3),(0.4)$, or $(0.5)$ below, with coefficients continuous in $\overline{\mathbb{R}_{+}^{n}}$, so that the corresponding $L$-harmonic measures on $\partial \mathbb{R}_{+}^{n}$ are singular with respect to the surface measure $\sigma$. In all four examples, first an operator $L_{x_{n-1}, x_{n}}$ is constructed on the half-plane $\left\{\left(x_{n-1}, x_{n}\right): x_{n}>0\right\}$, and then extended to $\mathbb{R}_{+}^{n}$ by adding the Laplacian of the other $(n-2)$ variables to become an operator in $\mathbb{R}_{+}^{n}$

$$
\sum_{1}^{n-2} \frac{\partial^{2}}{\partial x_{i}^{2}}+L_{x_{n-1}, x_{n}}
$$

and the corresponding $L$-harmonic measure is supported on a set of the form

$$
\mathbb{R}^{n-2} \times E \times\{0\}=\left\{x: x_{n-1} \in E, x_{n}=0\right\}
$$

for some $E \subseteq \mathbb{R}$ of zero length.

The techniques used by these authors are highly two dimensional. Therefore it is desirable to find a different approach which shall produce sets on $\partial \mathbb{R}_{+}^{n}$ more general than $(0.2)$, that have zero surface measure and positive $L$-harmonic measures for some $L$ on $\mathbb{R}_{+}^{n}$.

In this note, we succeed in finding a class of porous sets on $\partial \mathbb{R}_{+}^{n}$ which are perfect sets, can be described geometrically, and have all the properties mentioned above. The corresponding operators also cannot be built from any two dimensional operators in any obvious way. Moreover the porosity condition given is sharp.

Received by the editors October 14, 1992.

1991 Mathematics Subject Classification. Primary 31B35.

Partially supported by the National Science Foundation. 
The uniformly elliptic operators to be considered are

$$
L=\sum_{i, j=1}^{n} \frac{\partial}{\partial x_{i}}\left(A_{i j}(x) \frac{\partial}{\partial x_{j}}\right)
$$

with bounded measurable coefficients in $H \equiv \mathbb{R}_{+}^{n}$; or

$$
L=\sum_{i, j=1}^{n} A_{i j}(x) \frac{\partial^{2}}{\partial x_{i} \partial x_{j}}
$$

with bounded continuous coefficients in $H$. Here, $\left(A_{i j}\right)$ is symmetric and satisfies

$$
\lambda|\xi|^{2} \leq \lambda(x)|\xi|^{2} \leq \sum_{i, j=1}^{n} A_{i j}(x) \xi_{i} \xi_{j} \leq \Lambda(x)|\xi|^{2} \leq \lambda|\xi|^{2}
$$

for all $x \in H, \xi \in \mathbb{R}^{n}$, and some positive constants $\Lambda$ and $\lambda$.

Solutions of $L u=0$ are understood to be in the weak sense when $L$ is in the divergence form (0.3), and in the strong sense when $L$ is in the nondivergence form (0.4) ([9]). In view of the maximum principle, and the existence and the uniqueness of the solution for the Dirichlet problem ([7], [9]) there exists a unique positive Borel measure $\omega_{L}^{x}$ on $\partial H$ so that, for any $g \in C(\partial H)$,

$$
u(x)=\int_{\partial H} g(y) d \omega_{L}^{x}(y)
$$

is the solution of $L u=0$ in $H$, which is continuous on $\bar{H}$ and satisfies $u \mid \partial H=g$. And $\omega_{L}^{x}$ is called the $L$-harmonic measure at $x$.

The operators considered by Barcelo are in the form:

$$
L=\sum_{i, j=1}^{n} A_{i j}(x) \frac{\partial^{2}}{\partial x_{i} \partial x_{j}}+\sum_{i=1}^{n} B_{i}(x) \frac{\partial}{\partial x_{i}}
$$

where $\left(A_{i j}\right)$ is symmetric, uniformly elliptic, and continuous in $\bar{H}$, and $\left(B_{i}\right)$ satisfies additional conditions involving the modulus of continuity of $\left(A_{i j}\right)$ in the normal direction ([1], [2]).

Examples in [1] and [6] are based on a well-known theorem of Beurling and Ahlfors [4].

Theorem A. There exists a $K$-quasiconformal mapping of the upper half-plane $\mathbb{R}_{+}^{2}$ onto itself with the boundary correspondence $x \rightarrow h(x)$ if and only if $h$ is strictly increasing, $h(-\infty, \infty)=(-\infty, \infty)$, and

$$
\frac{1}{\rho} \leq \frac{h(x+t)-h(x)}{h(x)-h(x-t)} \leq \rho
$$

for some $\rho \geq 1$ and all real $x$ and $t$. Moreover $\rho$ determines a possible value of $K$ and vice versa.

Let $h$ be a singular function on $\mathbb{R}^{1}$ which satisfies $(0.6)$ and $f$ a quasiconformal extension of $h$ onto $\mathbb{R}_{+}^{2}$. Composition of $f$ with the Laplacian induces an operator $L$ in $\mathbb{R}_{+}^{2}$ of divergence form $(0.3)$, whose $L$-harmonic measure is singular with respect to the length. An operator in $H$ whose harmonic measure is supported on a set of the form (0.2) is obtained by adding the Laplacian of the other $(n-2)$ variables. This is the basic idea in [1] and [6]. 
The $n$-dimensional version of Theorem A ([5], [13]) states that a homeomorphism $h$ on $\mathbb{R}^{n-1}$ is the boundary correspondence of a $K$-quasiconformal mapping from $\mathbb{R}_{+}^{n}$ onto $\mathbb{R}_{+}^{n}(n \geq 3)$ if and only if $h$ itself is a quasiconformal mapping on $\mathbb{R}^{n-1}$. However, every quasiconformal mapping in $\mathbb{R}^{m}(m \geq 2)$ maps sets of Lebesgue measure zero to sets of Lebesgue measure zero. Moreover, the pullback of the Laplacian under a quasiconformal mapping in $\mathbb{R}^{n}$, $n \geq 3$, need not be uniformly elliptic. Therefore the method by quasiconformal mappings fails in $\mathbb{R}^{n}$ when $n \geq 3$.

The methods by Riesz products in [11] and [12] can conceivably be generalized to $\mathbb{R}_{+}^{n}$ to obtain $(n-1)$-dimensional sets on $\partial H$ general than $(0.2)$, which have zero $\sigma$-measure and positive $L$-harmonic measures for some $L$. Nonetheless the sets so obtained are dense and cannot be described geometrically.

Note that a homeomorphism $h$ on $\mathbb{R}^{1}$ satisfies $(0.6)$ if and only if the measure induced by $h$ has the doubling property; and recall that $\omega_{L}$-measures on $\partial H$ have the doubling property ([3], [7]).

Our approach is based on the relation between the porosity and the null sets of doubling measures ([15]).

Given $\left\{\alpha_{k}\right\}, 0<\alpha_{k}<1$, a set $F \subseteq \mathbb{R}^{m}$ is called $\left\{\alpha_{k}\right\}$-porous if there exists a sequence of coverings $\mathscr{C}_{k}=\left\{I_{k, j}\right\}$ of $F$ by cubes with mutually disjoint interiors, so that for every $k>k_{0}$, each $I_{k, j} \backslash F$ contains a cube $J_{k, j}$ of side length $l\left(J_{k, j}\right) \geq \alpha_{k} l\left(I_{k, j}\right)$, and that $\bigcup_{\mathscr{C}_{k+1}} I_{k+1, i}$ is contained in $\bigcup_{\mathscr{b}_{k}}\left(I_{k, j} \backslash J_{k, j}\right)$ and $\sup _{j} l\left(I_{k, j}\right) \rightarrow 0$ as $k \rightarrow \infty$.

Note that an $\left\{\alpha_{k}\right\}$-porous set has zero $m$-dimensional measure when $\sum \alpha_{k}^{m}=$ $\infty$. The Cantor ternary set on $\mathbb{R}$ is $\left\{\frac{1}{3}\right\}$-porous.

The following theorem is proved in [15].

Theorem B. Let $m \geq 1$ and $0<\alpha_{k}<\frac{1}{16}$. If

$$
\sum_{1}^{\infty} \alpha_{k}^{m K}=\infty \quad \text { for all } K \geq 1 \text {, }
$$

and $S \subseteq \mathbb{R}^{m}$ is $\left\{\alpha_{k}\right\}$-porous, then $S$ is a null set for all doubling measures on $\mathbb{R}^{m}$. If $\alpha_{k}$ is nonincreasing and satisfies

$$
\sum_{1}^{\infty} \alpha_{k}^{m K}<\infty \quad \text { for some } K \geq 1,
$$

then there exists an $\left\{\alpha_{k}\right\}$-porous set which carries a positive measure for some doubling measure on $\mathbb{R}^{m}$.

The analogous porosity and $\omega_{L}$-null set relation holds, despite the fact that not all doubling measures are $L$-harmonic measures.

Theorem 1. Let $n \geq 2$ and $0<\alpha_{k}<\frac{1}{16}$. If

$$
\sum_{1}^{\infty} \alpha_{k}^{(n-1) K}=\infty \text { for all } K \geq 1,
$$

and $S \subseteq \partial H$ is an $\left\{\alpha_{k}\right\}$-porous set in $\mathbb{R}^{n-1}$, then $S$ is $\omega_{L}$-null for all uniformly elliptic operators (0.3) and (0.4) on $H$.

This follows from Theorem B and the doubling property of $\omega_{L}$. 
The condition in Theorem 1 is sharp. Suppose that $\alpha_{n}$ is decreasing and that $\sum_{1}^{\infty} \alpha_{k}^{(n-1) K}<\infty$ for some $K \geq 1$. By Theorem B, there exists an $\left\{\alpha_{k}\right\}$-porous set $E$ on $\mathbb{R}^{1}$ which carries a positive $\mu$ measure for some doubling measure $\mu$ on $\mathbb{R}^{1}$. Thus $\mathbb{R}^{n-2} \times E \times\{0\}$ has positive $\omega_{L}$-measure for the divergence operator $L=\sum_{1}^{n-2} \frac{\partial^{2}}{\partial x_{i}^{2}}+L_{x_{n-1}, x_{n}}$ in $\mathbb{R}_{+}^{n}$; here $L_{x_{n-1}, x_{n}}$ is the pullback of the Laplacian under a two-dimensional quasiconformal mapping extension of $\mu$.

The purpose of this paper is to produce examples much more general than $\mathbb{R}^{n-2} \times E \times\{0\}$.

Given $\left\{\alpha_{k}\right\}, 0<\alpha_{k}<1$, and positive integers $\left\{M_{k}\right\}$, we say a set $S \subseteq \mathbb{R}^{m}$ is in the class $\Pi\left(\left\{\alpha_{k}\right\},\left\{M_{k}\right\}\right)$ if it can be constructed as follows. Let $\mathscr{C}_{k}=\left\{I_{k, j}\right\}$ be a finite collection of closed cubes in $\mathbb{R}^{m}$, :vith edges parallel to the coordinate axes, having mutually disjoint interiors and nearly common side lengths: $1 \leq$ $l\left(I_{1, j}\right) \leq 10$, and

$$
\prod_{1}^{k-1} \alpha_{i}^{M_{i}} \leq l\left(I_{k, j}\right) \leq 10 \prod_{1}^{k-1} \alpha_{i}^{M_{i}}, \quad k>1 .
$$

Denote $\bigcup_{\mathscr{C}_{k}} I$ by $S_{k}$, and require further that $S_{k+1}$ is obtained from $S_{k}$ by deleting the center $\alpha_{k}^{m}$ portion of each $I \in \mathscr{C}_{k}$ :

$$
S_{k+1}=S_{k} \backslash \bigcup\left\{\left(\alpha_{k} I\right)^{\circ}: I \in \mathscr{C}_{k}\right\}, \quad k \geq 1,
$$

where $\alpha_{k} I$ is the cube parallel and concentric to $I$ of side length $\alpha_{k} l(I)$, and $\left(\alpha_{k} I\right)^{\circ}$ denotes its interior. And $S=\bigcap_{1}^{\infty} S_{k}$.

Note that sets in this class are $\left\{\alpha_{k}\right\}$-porous and have some similarity to the Cantor sets. The fact that cubes in $\mathscr{C}_{k}$ are allowed to have different sizes enables us to produce many interesting sets. See $\S 4$.

Theorem 2. Let $n \geq 2$, and $0<\alpha_{k}<\frac{1}{16}$ be a decreasing sequence satisfying

$$
\sum_{1}^{\infty} \alpha_{k}^{(n-1) K}<\infty \quad \text { for some } K \geq 1 .
$$

Then on $\mathbb{R}^{n-1}$, any set in the class $\Pi\left(\left\{\alpha_{k}\right\},\left\{M_{k}\right\}\right)$ carries a positive $\omega_{L^{-}}$ measure for some operator (0.3) (and some operator (0.4)) provided that $M_{k}$ are all larger than a constant $M(n, K)$. Moreover, the ellipticity ratio $\Lambda / \lambda$ of this operator has an upper bound $C(n, K)$ which approaches 1 as $K \rightarrow 1$.

Theorem 3. Let $n \geq 2$, and $\left\{\alpha_{k}\right\}$ and $\left\{K_{k}\right\}$ be two decreasing sequences with $0<\alpha_{k}<\frac{1}{16}, \lim \alpha_{k}=0, K_{k}>1$, and $\lim K_{k}=1$. Assume further that

$$
\sum \alpha_{k}^{n-1}=\infty, \quad \sum \alpha_{k}^{(n-1) K_{k}}<\infty .
$$

Then on $\mathbb{R}^{n-1}$, any set in the class $\Pi\left\{\left(\alpha_{k}\right\},\left\{M_{k}\right\}\right)$ carries a positive $\omega_{L^{-}}$ measure for some operator (0.3) (and an operator (0.4)) provided that $M_{k}$ are all larger than a constant $M(n)$. Moreover, the coefficients $\left(A_{i j}(x)\right)$ can be chosen to be continuous on $\bar{H}$, with $\left\|A_{i j}(x)-\delta_{i j}\right\|_{\infty} \rightarrow 0$ uniformly as $x \rightarrow \partial H$, and the ellipticity parameters satisfy $\Lambda(x) / \lambda(x) \rightarrow 1$ uniformly as $x \rightarrow \partial H$.

Furthermore, we prove in $\S 4$ the following. 
Theorem 4. Assume that $0<\alpha_{k}<\frac{1}{16}$ is decreasing, $\sum \alpha_{k}^{n-1}=\infty$ and that $(0.8)$ or $(0.9)$ is satisfied. Then there are $\left\{\alpha_{k}\right\}$-porous sets on $\partial H$ which meet "every" ( $n-2)$-dimensional hyperplane $\left\{x: x_{n}=0, x_{j}=a\right\}, 1 \leq j \leq n-1$ and $a \in \mathbb{R}$, on a set of zero $(n-2)$-dimensional measure, but carry a positive $\omega_{L}$-measure for some operator (0.3) (or some operator (0.4)). As before the ellipticity parameters of $L$ can be chosen to depend on $n$ and $K$ only in the case $(0.8)$, and the coefficients of $L$ can be chosen to be continuous on $\bar{H}$ with ellipticity $\Lambda(x) / \lambda(x) \rightarrow 1$ uniformly as $x \rightarrow \partial H$ in the case (0.9).

The author is indebted to J. Heinonen and M. Zinsmeister for pointing out an earlier error in the construction of the operator $L_{1}$ in $\S 2$. An alternative construction of $L_{1}$ based on a suggestion of Heinonen is given at the end of that section.

\section{Preliminaries}

Let $L$ be an operator in the form (0.3) or (0.4). We refer the reader to [3], [7], [8], and [9], for the maximum principle, the existence and uniqueness of the Dirichlet problem in certain domains, and the lemmas listed below.

Denote by $\omega_{L}^{x}(E, D)$ the bounded solution of $L=0$ in a domain $D$ with boundary value 1 on $E \subseteq \partial D$ and 0 on $\partial D \backslash \bar{E}$, if indeed it exists uniquely. For the half-space $H$, this solution $\omega_{L}^{x}(E, H)$ agrees with the previously defined $L$-harmonic measure $\omega_{L}^{x}(E)$.

Denote by $B(y, r)=\{x:|x-y|<r\}$, and $\Delta(y, r)=B(y, r) \cap \partial H$. Denote by $C$ and $c$ positive constants which depend only on the quantities in parentheses, and whose values may vary from line to line.

Lemma 1. There exists $C(n, \Lambda / \lambda)>0$ so that (1.1) $\omega_{L}^{x}(\Delta(0, \rho), H \cap B(0, \rho)) \geq C(n, \Lambda / \lambda) \quad$ for all $x \in H \cap B(0, \rho / 2)$.

Lemma 2 (Harnack Principle). There exists $C(n, \Lambda / \lambda)>0$ so that if $u$ is a positive solution of $L=0$ in $B(x, 2 r)$, then

$$
\sup _{B(x, r)} u \leq C(n, \Lambda / \lambda) \inf _{B(x, r)} u .
$$

Lemma 3 (Doubling Property). Let $y \in \partial H$ and $\rho>0$. Then

$$
\omega_{L}^{x}(\Delta(y, 2 \rho), H) \leq C(n, \Lambda / \lambda) \omega_{L}^{x}(\Delta(y, \rho), H)
$$

for all $x \in H \backslash B(y, 4 \rho)$.

Lemma 4. There exist constants $\beta=\beta(n, \Lambda / \lambda)$ and $\gamma=\gamma(n, \Lambda / \lambda), 0<$ $\beta, \gamma<1$, so that if $x \in H$ and $|x|>2 \rho>0$, then

$$
\omega_{L}^{x}(\Delta(0, \rho), H) \leq\left(\frac{\rho}{|x|}\right)^{\beta} ;
$$

and if $x \in H$ and $|x|<\rho / 2$, then

$$
\omega_{L}^{x}(\partial B(0, \rho) \cap H, B(0, \rho) \cap H)<\left(\frac{|x|}{\rho}\right)^{\gamma} .
$$

Moreover, $\beta(n, \Lambda / \lambda)$ may be chosen to be a decreasing function of $\Lambda / \lambda$.

The estimate (1.3) follows from the doubling property, and the inequality (1.4) is obtained by iterating (1.1). 
Lemma 5 (Boundary Harnack Principle). Let $u$ and $v$ be two positive solutions of $L=0$ in $B(0, R) \cap H$ which vanish continuously on $\Delta(0, R)$. Then

$$
C(n, \Lambda / \lambda)^{-1} \frac{u((0, \ldots, 0, R / 2))}{v((0, \ldots, 0, R / 2))} \leq \frac{u(x)}{v(x)} \leq C(n, \Lambda / \lambda) \frac{u((0, \ldots, 0, R / 2))}{v((0, \ldots, 0, R / 2))}
$$

for all $x \in B(0, R / 2) \cap H$.

\section{OPERATORS IN DIVERGENCE FORM}

In this section, we prove Theorems 2 and 3 in the divergence case.

Let $r=|x|$ for $x \in \mathbb{R}^{n}, \Delta$ be the Laplace's operator, and $\delta$ be the Beltrami operator in the spherical coordinates. Then

$$
\Delta=\frac{\partial^{2}}{\partial r^{2}}+(n-1) r^{-1} \frac{\partial}{\partial r}+r^{-2} \delta .
$$

Let $b>0$, and

$$
L_{1}=\frac{\partial^{2}}{\partial r^{2}}+(n-1) r^{-1} \frac{\partial}{\partial r}+b r^{-2} \delta
$$

Rewrite the operator to obtain

$$
\begin{aligned}
L_{1}= & b \Delta-(b-1) \frac{\partial^{2}}{\partial r^{2}}-(b-1)(n-1) r^{-1} \frac{\partial}{\partial r} \\
= & b \sum_{i} \frac{\partial^{2}}{\partial x_{i}^{2}}-(b-1) \sum x_{i} x_{j}|x|^{-2} \frac{\partial^{2}}{\partial x_{i} \partial x_{j}} \\
& -(b-1)(n-1)|x|^{-1} \sum_{i} x_{i}|x|^{-1} \frac{\partial}{\partial x_{i}} .
\end{aligned}
$$

This operator can also be expressed in the divergence form:

$$
L_{1}=\sum \frac{\partial}{\partial x_{i}}\left(A_{i j} \frac{\partial}{\partial x_{j}}\right)
$$

where

$$
A_{i j}(x)=b \delta_{i j}-(b-1) x_{i} x_{j}|x|^{-2},
$$

and $\delta_{i j}=1$ if $i=j$, and $=0$ if $i \neq j$. The coefficient matrix $A(x)$ is symmetric and has eigenvalues $1, b, b, \ldots, b$.

Differentiation indicates that $|x|^{-n+2},(-\log |x|, n=2), x_{n}|x|^{-d}$, and $x_{n}|x|^{-d^{\prime}}$ are solutions of $L_{1}=0$ in $\mathbb{R}^{n} \backslash\{0\}$, where

$$
d=\left(n+\left(n^{2}+4(n-1)(b-1)\right)^{1 / 2}\right) / 2,
$$

and

$$
d^{\prime}=\left(n-\left(n^{2}+4(n-1)(b-1)\right)^{1 / 2}\right) / 2 .
$$

And they are the Green function with pole at 0 for $\mathbb{R}^{n}$, and the kernel functions on $H$ with poles at 0 and $\infty$ respectively.

We shall give the harmonic measure estimate which leads to our construction. It is important to note that $d>n$ when $b>1$. 
Proposition 1. Let $D=H \cap B(0, R), x \in D, 0<\rho<R$. Then

$$
\omega_{L_{1}}^{\times}(\Delta(0, \rho), D) \leq C(n, b)\left(\frac{\rho}{|x|}\right)^{d-1} .
$$

Proof. We assume, as we may, that $0<\rho<|x| / 4$. Let $u(x)=x_{n}|x|^{-d}$, and $T=T_{1} \cup T_{2}$ where

$$
T_{1}=\left\{x: x_{1}^{2}+x_{2}^{2}+\cdots+x_{n-1}^{2}=4 \rho^{2}, 0<x_{n}<\rho\right\},
$$

and

$$
T_{2}=\left\{x: x_{1}^{2}+x_{2}^{2}+\cdots+x_{n-1}^{2} \leq 4 \rho^{2}, x_{n}=\rho\right\} .
$$

We claim that

$$
\omega_{L_{1}}^{x}(\Delta(0, \rho), D) \leq c(n, b) \rho^{d-1} u(x) \quad \text { on } T .
$$

This is clear for $x \in T_{2}$. Let $x \in T_{1}$ and $\tilde{x}=\left(x_{1}, \ldots, x_{n-1}, \rho\right)$. In view of Lemma 1, the boundary Harnack principle and the Harnack principle, we have

$$
\frac{\omega_{L_{1}}^{x}(\Delta(0, \rho))}{u(x)} \cong \frac{\omega_{L_{1}}^{\tilde{x}}(\Delta(0, \rho), D)}{u(\tilde{x})} \cong \frac{\omega_{L_{1}}^{(0, \ldots, 0, \rho)}(\Delta(0, \rho), D)}{u((0, \ldots, 0, \rho))} \cong \rho^{d-1}
$$

by $\cong$ we mean the ratio of two sides is bounded above and below by some positive constants depending on $n$ and $b$ only.

Finally (2.2) follows from the claim and the maximum principle.

Remark 1. If $n \geq 3$ and $L$ is a divergence operator $(0.3)$ in $H$, then

$$
\omega_{L}^{(0, \ldots, 0,1)}(\Delta(0, \rho), H) \leq C(n, \Lambda / \lambda) \rho^{n-2}, \quad 0<\rho<1 .
$$

This follows from the fact that the Green function $G_{L}(x, y)$ is asymptotic to $|x-y|^{2-n}$ near the pole $x$ for every divergence operator (0.3) ([10]).

The operator $L_{1}$ shows that the exponent $n-2$ in the harmonic measure estimation is sharp, and that an analogous lower estimate for all operators $(0.3)$ does not exist. In fact, the $L_{1}$-harmonic measure of $\Delta(0, \rho)$ is comparable to $\rho^{d-1}$, with $d$ given in $(2.1)$ :

$$
c(n, b) \rho^{d-1} \leq \omega_{L_{1}}^{(0, \ldots, 0,1)}(\Delta(0, \rho), H) \leq C(n, b) \rho^{d-1}, \quad 0<\rho<1 .
$$

The upper estimate is $(2,2)$ when $R \rightarrow \infty$; the lower estimate follows easily from (1.1) and the maximum principle. Note from (2.1) that $d-1>n-2$, and that $d-1$ approaches $n-2$ as $b \rightarrow 0^{+}$, and it approaches $\infty$ as $b \rightarrow \infty$.

Assume, from now on, that $b>1$; thus $d>n$ and $L_{1}$ repels a stochastic process from the origin. We shall place translates of $L_{1}$ in a sequence of hemispheres accumulating on $\partial H$.

Let $S \subseteq \partial H$ be a set in the class $\Pi\left(\left\{\alpha_{k}\right\},\left\{M_{k}\right\}\right)$ with $\left\{M_{k}\right\}$ to be specified later, $\mathscr{C}_{k}=\left\{I_{k, j}\right\}$ be the cubes associated with $S$ in its construction. We shall construct an operator $L_{2}$ so that

$$
\omega_{L_{2}}^{(0, \ldots, 0,1)}\left(\alpha_{k} I_{k, j}\right) \leq C(n, K) \alpha_{k}^{(n-1) K} \omega_{L_{2}}^{(0, \ldots, 0,1)}\left(I_{k, j}\right)
$$

for sufficiently large $k$ 's.

Let

$$
l_{k}=\prod_{1}^{k} \alpha_{j}^{M_{j}} \quad(1 \leq k<\infty)
$$


and recall that

$$
l_{k-1} \leq l\left(I_{k, j}\right) \leq 10 l_{k-1} .
$$

Denote by $Y_{k}$ the collection of centers of cubes in $\mathscr{C}_{k}$, and

$$
\mathbb{Z}_{k}=\left\{\left(y, l_{k}\right): y \in Y_{k}\right\} \text {. }
$$

To each $w \in \mathbb{Z}_{k}$, we define

$$
p(w)=B\left(w, l_{k-1} / 4\right) \cap\left\{x: x_{n}>l_{k}\right\},
$$

and

$$
q(w)=B\left(w, l_{k-1} / 3\right) \cap\left\{x: x_{n}>\frac{9}{10} l_{k}\right\} .
$$

Let $b(x)$ be a function smooth in $H, 1 \leq b(x) \leq b$, and

$$
b(x)= \begin{cases}b, & \text { on } \bigcup_{k} \bigcup_{w \in \mathbb{Z}_{k}} p(w), \\ 1, & \text { on } H \backslash \bigcup_{k} \bigcup_{w \in \mathbb{Z}_{k}} q(w) .\end{cases}
$$

Define in $H$,

$$
A_{i j}(x)=\left\{\begin{aligned}
b(x) \delta_{i j}-(b(x)-1)\left(x_{i}-w_{i}\right)\left(x_{j}-w_{j}\right)|x-w|^{-2}, \\
\quad \text { if } x \in q(w) \backslash\{w\} \text { for some } w \in \mathbb{Z}_{k}(k \geq 1), \\
\delta_{i j}, \quad \text { if } x \in\left(H \backslash \bigcup_{k} \bigcup_{w \in \mathbb{Z}_{k}} q(w)\right) \cup \bigcup_{k} \mathbb{Z}_{k},
\end{aligned}\right.
$$

and

$$
L_{2}(x)=\sum_{i, j} \frac{\partial}{\partial x_{i}}\left(A_{i j}(x) \frac{\partial}{\partial x_{j}}\right) .
$$

In each $p(w), L_{2}$ is a translate of $L_{1}$, thus a translated version of Proposition 1 holds for $L_{2}$ in $p(w)$. Clearly $A(x)$ is smooth in $H \backslash \bigcup_{k} \mathbb{Z}_{k}$, and it satisfies

$$
|\xi|^{2} \leq \sum A_{i j}(x) \xi_{i} \xi_{j} \leq b|\xi|^{2}
$$

and

$$
\left\|A_{i j}(x)-\delta_{i j}\right\|_{\infty} \leq b-1
$$

for all $x \in H$ and $\xi \in \mathbb{R}^{n}$.

To each $w \in \mathbb{Z}_{k}$, define

$$
t(w)=B\left(w, 10 \sqrt{n} \alpha_{k} l_{k-1}\right) \cap\left\{x: x_{n}=l_{k}\right\},
$$

and note that $t(w) \subseteq \overline{p(w)}$ if $k$ is sufficiently large.

For a set $\eta$ in $H$, denote by $\tilde{\eta}=\left\{\left(x_{1}, x_{2}, \ldots, x_{n-1}, 0\right):\left(x_{1}, x_{2}, \ldots, x_{n}\right) \in\right.$ $\eta\}$ its projection onto $\partial H$; for a half-ball $\eta$ in $H$, denote by $\hat{\eta}$ the spherical part of the boundary of $\eta$.

We now given the main estimation in the proof.

Lemma 6. Let $\beta=\beta(n, b)$ and $\gamma=\gamma(n, b)$ be the constants chosen in Lemma 4 , and let $0<\tau<1, H_{k}=\left\{x: x_{n}>l_{k}\right\}$, and $w \in \mathbb{Z}_{k}$. Then

$$
\omega_{L_{2}}^{x}\left(t(w), H_{k}\right) \leq c(n, b) \alpha_{k}^{(1-\tau)(d-1)+\tau \beta}, \quad x \in H_{k} \backslash p(w),
$$

provided that $k>C\left(n, \tau, b,\left\{\alpha_{k}\right\}\right)$.

Proof. Let $s(w)=B\left(w, \alpha_{k}^{\tau} l_{k-1}\right) \cap\left\{x: x_{n}>l_{k}\right\}$. Consider only large $k$ 's with $\alpha_{k}<\min \left\{n^{-1}, 4^{-1 / \tau}\right\}$; thus $t(w) \subseteq \overline{s(w)} \subset \overline{p(w)}$. It suffices to verify (2.6) for $x \in \hat{p}(w)$ only. Denote $\omega_{L_{2}}^{x}$ by $\omega^{x}$ for simplicity. 
Given $x \in \hat{p}(w)$, it follows from the maximum principle that

$$
\omega^{x}\left(t(w), H_{k}\right) \leq \omega^{x}\left(\hat{s}(w), H_{k} \backslash \overline{s(w)}\right) \sup _{y \in \hat{s}(w)} \omega^{y}\left(t(w), H_{k}\right) .
$$

From (1.1), (1.2), (1.3), and the maximum principle, it follows that

$$
w^{x}\left(\hat{s}(w), H_{k} \backslash \overline{s(w)}\right) \leq C(n, b) \alpha_{k}^{\tau \beta} \quad \text { for } x \in \hat{p}(w) ;
$$

from (1.4) and Proposition 1, it follows that for $y \in \hat{s}(w)$,

$$
\begin{aligned}
\omega^{y}\left(t(w), H_{k}\right) & \leq \omega^{y}(t(w), p(w))+\omega^{y}(\hat{p}(w), p(w)) \sup _{v \in \hat{p}(w)} \omega^{x}\left(t(w), H_{k}\right) \\
& \leq C(n, b)\left\{\left(\frac{\sqrt{n} \alpha_{k}}{\alpha_{k}^{\tau}}\right)^{d-1}+\left(\alpha_{k}^{\tau}\right)^{\gamma} \sup _{x \in \hat{p}(w)} \omega^{x}\left(t(w), H_{k}\right)\right\} .
\end{aligned}
$$

For sufficiently large $k$ 's satisfying $C(n, b) \alpha_{k}^{\tau \gamma}<\frac{1}{2}$ in (2.7), we obtain (2.6).

An estimation similar to (2.6) holds for the projection of $t(w)$ on $\partial H$ provided that $k$ is sufficiently large.

Lemma 7. Let $0<\tau<1, w \in \mathbb{Z}_{k}$, and

$$
r(w)=\hat{p}(w) \cup\left\{x:\left(\sum_{1}^{n-1}\left(x_{j}-w_{j}\right)^{2}\right)^{1 / 2}=l_{k-1} / 4,0<x_{n} \leq l_{k}\right\} .
$$

Then

$$
\omega_{L_{2}}^{x}(\tilde{t}(w)) \leq C(n, b)\left\{\alpha_{k}^{(1-\tau)(d-1)+\tau \beta}+\alpha_{k}^{\left(M_{k}-1\right) \gamma}\right\}
$$

for $x \in r(w)$, provided that $k>C\left(n, b, \tau,\left\{\alpha_{k}\right\}\right)$.

Proof. Again let $\omega^{x}=\omega_{L_{2}}^{x}$. If $x \in r(w) \backslash \hat{p}(w)$, then $x_{n} \leq l_{k}$ and $\operatorname{dist}\left(x, \frac{1}{2} \tilde{t}(w)\right)$ $>\frac{1}{8} l_{k-1}$. Thus by (1.4), (2.8), and the maximum principle, we obtain

$$
\omega^{x}\left(\frac{1}{2} \tilde{t}(w)\right) \leq C(n, b) \alpha_{k}^{M_{k} \gamma} .
$$

If $x \in \hat{p}(w)$, then for sufficiently large $k$,

$$
\begin{aligned}
\omega^{x}\left(\frac{1}{2} \tilde{t}(w)\right) & \leq \omega^{x}\left(t(x), H_{k}\right)+\sup _{y \in \partial H_{k} \backslash t(w)} \omega^{y}\left(\frac{1}{2} \tilde{t}(w)\right) \\
& \leq C(n, b) \alpha_{k}^{(1-\tau)(d-1)+\tau \beta}+C(n, b) \alpha_{k}^{\left(M_{k}-1\right) \gamma} .
\end{aligned}
$$

The last estimate follows from (1.4) and the facts that $\operatorname{dist}\left(y, \frac{1}{2} \tilde{t}(w)\right) \geq \frac{1}{4} \alpha_{k} l_{k-1}$ and $y_{n}=l_{k}=\alpha_{k}^{M_{k}} l_{k-1}$ for $y \in \partial H_{k} \backslash t(w)$. Finally, (2.8) follows from the doubling property of $L_{2}$.

To complete the proof of Theorem 2, we need to choose $b$ and $M_{k}$ depending on $n$ and $K$ only so that

$$
(1-\tau)(d-1) \geq(n-1) K \quad \text { and } \quad\left(M_{k}-1\right) \gamma \geq(n-1) K
$$

and that

$$
b \rightarrow 1^{+} \text {as } K \rightarrow 1^{+} \text {. }
$$

The second requirement allows $L_{2}$ to approach $\Delta$ as $K \rightarrow 1$. For this purpose we may choose $b=1+4(n-1)\left(K^{4}-1\right), \tau=1-K^{-1}$, and $\gamma=\gamma(n, b)$, and let

$$
M_{k} \geq 3+n K \gamma^{-1}
$$


More efficient choices of these constants would depend strongly on good estimates of $\beta(n, \Lambda / \lambda)$ and $\gamma(n, \Lambda / n)$ in Lemma 4 , which have not been obtained.

For each $w \in \mathbb{Z}_{k}$, let $\tilde{w}=\left(w_{1}, w_{2}, \ldots, w_{n-1}, 0\right), I(w)$ be the $(n-1)$ dimensional cube in $\mathscr{C}_{k}$ centered at $\tilde{w}$, and $J(w)=\alpha_{k} I(w)$.

Lemma 8. Let $P=(0,0, \ldots, 0,1)$ and $w \in \mathbb{Z}_{k}$. Then

$$
\omega_{L_{2}}^{P}(J(w)) / \omega_{L_{2}}^{P}(I(w)) \leq C(n, K) \alpha_{k}^{(n-1) K},
$$

provided that $k>C\left(n, K,\left\{\alpha_{k}\right\}\right)$.

Proof. Recall from (0.7) that $l(J(w))=\alpha_{k} l(I(w)) \leq 10 \alpha_{k} l_{k-1}$. Therefore $J(w) \subseteq \tilde{t}(w)$, and it follows from (2.8) and (2.9) that for sufficiently large $k$,

$$
\omega_{L_{2}}^{x}(J(w)) \leq C(n, K) \alpha_{k}^{(n-1) K}, \quad x \in r(w) .
$$

Also from (1.1) and (1.2) it follows that

$$
\omega_{L_{2}}^{x}(I(w)) \geq C(n, K), \quad x \in r(w) .
$$

Thus (2.10) follows from the maximum principle.

From (2.10), the construction of $S$ and the hypothesis $\sum \alpha_{k}^{(n-1) K}<\infty$, it follows that for a sufficiently large $k_{0}$,

$$
\omega_{L_{2}}^{P}(S) \geq \omega_{L_{2}}^{P}\left(\bigcup_{\mathbb{Z}_{1}} I(w) \backslash \bigcup_{k=1}^{k_{0}-1} \bigcup_{\mathbb{Z}_{k}} J(w)\right) \prod_{k \geq k_{0}}\left(1-C(n, K) \alpha_{k}^{(n-1) K}\right)>0 .
$$

This completes the proof of Theorem 2 for the divergence case.

Proof of Theorem 3. We may assume that $\alpha_{k}$ and $K_{k}$ satisfy, in addition to (0.9), that

$$
\alpha_{k}^{K_{k}-1} \rightarrow 0 \quad \text { as } k \rightarrow \infty .
$$

In fact, there exists a positive sequence $\left\{\varepsilon_{k}\right\}, \lim \varepsilon_{k}=0$, such that $\lim \varepsilon_{k} \log \alpha_{k}$ $=-\infty$. Replacing $K_{k}$ by $K_{k}+\varepsilon_{k}$ if necessary, we obtain (2.11).

Let $\gamma=\gamma(n, 4)$ be the constant in Lemma 4,

$$
b_{k}=1+4(n-1)\left(K_{k}^{4}-1\right),
$$

and

$$
M_{k} \geq 3+2 n \gamma^{-1} \text {. }
$$

Let $S$ be a set on $\partial H$ in the class $\Pi\left(\left\{\alpha_{k}\right\},\left\{M_{k}\right\}\right)$, and retain all notations $\mathscr{C}_{k}=\left\{I_{k, j}\right\}, l_{k}, \mathbb{Z}_{k}, H_{k}, p(w), t(w), r(w), I(w)$, and $J(w)$ associated with $S$ from the proof of Theorem 2 .

In place of (2.4), choose a new $b(x)$, smooth in $H$, continuous on $\bar{H}$ and satisfying

$$
1 \leq b(x) \leq b_{k} \quad \text { on }\left\{x: l_{k} / 2 \leq x_{n} \leq l_{k-1} / 2\right\},
$$

and

$$
b(x)= \begin{cases}b_{k}, & \text { on } \bigcup_{w \in \mathbb{Z}_{k}} p(w)(k \geq 1), \\ 1, & \text { on } H \backslash \bigcup_{k} \bigcup_{w \in \mathbb{Z}_{k}} q(w) .\end{cases}
$$


Let $A(x)$ be the coefficient matrix (2.5) associated with this new $b(x)$, and $L_{3}=\sum \frac{\partial}{\partial x_{i}}\left(A_{i j}(x) \frac{\partial}{\partial x_{j}}\right)$ be the corresponding divergence operator. As before, in $\left\{x: l_{k} / 2 \leq x_{k} \leq l_{k-1} / 2\right\}$,

$$
|\xi|^{2} \leq \sum A_{i j}(x) \xi_{i} \xi_{j} \leq b_{k}|\xi|^{2}
$$

and

$$
\left\|A_{i j}(x)-\delta_{i j}\right\|_{\infty} \leq b_{k}-1
$$

Note also that $A_{i j}(x)$ are continuous in $\bar{H}$ except at $\bigcup_{k} \mathbb{Z}_{k}$.

Lemma 9. If $k$ is sufficiently large and $w \in \mathbb{Z}_{k}$ then

$$
\omega_{L_{3}}^{x}\left(t(w), H_{k}\right) \leq C(n) \alpha_{k}^{(n-1) K_{k}}, \quad x \in H_{k} \backslash p(w) .
$$

Proof. Fix $k$, let $\tau=1-K_{k}^{-1}$, and follow the proof of Lemma 6. We deduce from (2.12) that for $x \in \hat{p}(w)$ and sufficiently large $k$,

$$
\omega_{L_{3}}^{x}\left(t(w), H_{k}\right) \leq C(n)\left\{\alpha_{k}^{(n-1) K_{k}}+\alpha_{k}^{\gamma\left(1-K_{k}^{-1}\right)} \sup _{x \in \hat{p}(w)} \omega^{x}\left(t(x), H_{k}\right)\right\} .
$$

In view of (2.11), $C(n) \alpha_{k}^{\gamma\left(1-K_{k}^{-1}\right)} \rightarrow 0$ as $k \rightarrow \infty$, whence (2.13) follows.

Lemma 10. Let $P=(0,0, \ldots, 0,1), w \in \mathbb{Z}_{k}$, and $r(w), I(w)$, and $J(w)$ be the sets defined in Lemmas 7 and 8 associated with the current choices of $M_{k}$ 's. Then for large $k$,

$$
\omega_{L_{3}}^{x}(\tilde{t}(w)) \leq C(n) \alpha_{k}^{(n-1) K_{k}}, \quad x \in r(w),
$$

and

$$
\omega_{L_{3}}^{P}(J(w)) / \omega_{L_{3}}^{P}(I(w)) \leq C(n) \alpha_{k}^{(n-1) K_{k}} .
$$

Proof follows from that of Lemmas 7 and 8 with $\tau=1-K_{k}^{-1}$.

Thus, we may conclude from the hypothesis $(0.9)$ and the construction of $S$ that $S$ has zero $\sigma$-measure and positive $L_{3}$-harmonic measure.

All assertions in. Theorem 3 are now fulfilled, except that $\left(A_{i j}(x)\right)$ are still discontinuous at $\bigcup_{k} \mathbb{Z}_{k}$. Modify $\left(A_{i j}(x)\right)$ near each $w \in \mathbb{Z}_{k}$ so that in each $q(w)$

$$
A_{i j}(x)=b(x) \delta_{i j}-a_{k}(|x-w|)(b(x)-1)\left(x_{i}-w_{i}\right)\left(x_{j}-w_{j}\right)|x-w|^{-2},
$$

for some continuous $a_{k}(\rho)$ on $[0,1]$, with $0 \leq a_{k}(\rho) \leq 1, a_{k}(0)=0$, and $a_{k}(\rho)=1$ for $\rho \geq \rho_{k}>0$. This change of coefficients will affect the above harmonic measure estimations by at most a multiplicative constant, provided that $\rho_{k} \ll \alpha_{k} l_{k-1}$. This completes the proof of Theorem 3 for divergence operators.

We now give an alternative construction of $L_{1}$ following a suggestion of $\mathrm{J}$. Heinonen.

For $x \in \mathbb{R}^{n}$, denote $\left(x_{n-1}, x_{n}\right)$ by $z$. Let $\Phi$ be the quasiconformal mapping on $\mathbb{R}^{2}$ defined by

$$
\Phi(z)=z|z|^{\gamma} \quad(\gamma>0) .
$$

Let $a(z)=\left(\Phi^{\prime}(z)^{T}\right)^{-1}\left|\operatorname{det} \Phi^{\prime}(z)\right|\left(\Phi^{\prime}(z)\right)^{-1}$,

$$
L_{0}=\sum_{i, j=n-1}^{n} \frac{\partial}{\partial x_{i}}\left(a_{i j}(z)\right) \frac{\partial}{\partial x_{j}}
$$


and

$$
\tilde{L}_{1}=L_{0}+\sum_{1}^{n-2} \frac{\partial^{2}}{\partial x_{i}^{2}}
$$

It is easy to check that the ratio of ellipticity parameters is bounded above and below by $1+\gamma$ and 1 . In place of (2.2), we have

Proposition $1^{\prime}$. Let $D=H \cap B(0, R), x \in D, 0<\rho<R$. Then

$$
\omega_{\widetilde{L}_{1}}^{x}(\Delta(0, \rho), D) \leq C(n, \gamma)\left(\frac{\rho}{|x|}\right)^{1+\gamma} .
$$

Proof. Assume as before that $0<\rho<|x| / 4$. It follows from Theorem 1.1 in [7] and the Harnack Principle that

$$
\begin{aligned}
\omega_{\widetilde{L}_{1}}^{x}(\Delta(0, \rho), D) & \leq C(n, \gamma) \omega_{\widetilde{L}_{1}}^{(0, \ldots, 0,|x|)}(\Delta(0, \rho), D) \\
& \leq C(n, \gamma) \omega_{\widetilde{L}_{1}}^{(0, \ldots, 0,|x|)}\left(\Delta(0, \rho), \mathbb{R}_{+}^{n}\right) \\
& \leq C(n, \gamma) \omega_{\widetilde{L}_{1}}^{(0, \ldots, 0,|x|)}\left(T(\rho), \mathbb{R}_{+}^{n}\right)
\end{aligned}
$$

where $T(\rho)=\left\{\left(x_{1}, x_{2}, \ldots, x_{n-1}, 0\right):\left|x_{n-1}\right|<\rho\right\}$. Note that if $L_{0} u=0$ and $\tilde{u}(x)=u\left(x_{n-1}, x_{n}\right)$ then $\tilde{u}$ is a solution of $\widetilde{L}_{1}=0$. Therefore

$$
\omega_{\widetilde{L}_{1}}^{(0, \ldots, 0,|x|)}\left(T(\rho), \mathbb{R}_{+}^{n}\right)=\omega_{L_{0}}^{(0,|x|)}\left(I(\rho), \mathbb{R}_{+}^{2}\right)
$$

where $I(\rho)=\left\{\left(x_{n-1}, 0\right):\left|x_{n-1}\right|<\rho\right\}$. In $\mathbb{R}^{2}$, it is known that

$$
\begin{aligned}
\omega_{L_{0}}^{(0,|x|)}\left(I(\rho), \mathbb{R}_{+}^{2}\right) & =\omega_{\Delta}^{\Phi(0,|x|)}\left(I(|\Phi(0, \rho)|), \mathbb{R}_{+}^{2}\right) \\
& \leq C \frac{|\Phi(0, \rho)|}{|\Phi(0,|x|)|}=C\left(\frac{\rho}{|x|}\right)^{1+\gamma} .
\end{aligned}
$$

This completes the proof of Proposition $1^{\prime}$. And $\widetilde{L}_{1}$ may be used in place of $L_{1}$ in constructing $L_{2}$ and the porous sets.

\section{OPERATORS IN NONDIVERGENCE FORM}

Let $b>0$, and

$$
L_{4}=\frac{\partial^{2}}{\partial r^{2}}+b(n-1) r^{-1} \frac{\partial}{\partial r}+b r^{-2} \delta .
$$

Then $|x|^{-b(n-1)+1}(-\log |x|$, if $b(n-1)=1), x_{n}|x|^{-b(n-1)-1}$, and $x_{n}$ are solutions of $L_{4}=0$ in $\mathbb{R}^{n} \backslash\{0\}$. Rewrite the operator to obtain

$$
L_{4}=b \Delta-(b-1) \frac{\partial^{2}}{\partial r^{2}}=b \sum_{i} \frac{\partial^{2}}{\partial x_{i}^{2}}-(b-1) \sum_{i, j} x_{i} x_{j}|x|^{-2} \frac{\partial^{2}}{\partial x_{i} \partial x_{j}} .
$$

The coefficient matrix $\left(A_{i j}(x)\right)$ is symmetric and has eigenvalues $1, b, b, \ldots$, $b$.

Proposition 2. Let $D=H \cap B(0, R), x \in D, 0<\rho<R$. Then

$$
\omega_{L_{4}}^{x}(\Delta(0, \rho), D) \leq C(n, b)\left(\frac{\rho}{|x|}\right)^{(n-1) b} .
$$


Proof. Instead of applying the boundary Harnack Principle as in Proposition 1 , we give a conceptually simpler proof based on an auxiliary function $v(x)$. We assume, as we may, that $0<\rho<|x| / 4$. Let $u(x)=x_{n}|x|^{-b(n-1)-1}$, and $T=T_{1} \cup T_{2}$ where $T_{1}=\left\{x: x_{1}^{2}+x_{2}^{2}+\cdots+x_{n-1}^{2}=4 \rho^{2}, 0<x_{n}<\rho\right\}$ and $T_{2}=\left\{x: x_{1}^{2}+x_{2}^{2}+\cdots+x_{n-1}^{2} \leq 4 \rho^{2}, x_{n}=\rho\right\}$. We claim that

$$
\omega_{L_{4}}^{x}(\Delta(0, \rho), D) \leq C(n, b) \rho^{b(n-1)} u(x) \quad \text { on } T .
$$

Fix a point $a$ on $\partial H$ with $a_{1}^{2}+\cdots+a_{n-1}^{2}=4 \rho^{2}$, and define as in [3, p. 159],

$$
v(x)=1-\left(\frac{\rho^{2}}{\left(x_{1}-a_{1}\right)^{2}+\left(x_{2}-a_{2}\right)^{2}+\cdots+\left(x_{n-1}-a_{n-1}\right)^{2}+\left(x_{n}+\rho\right)^{2}}\right)^{n b} .
$$

Following Bauman [3], we deduce that $L_{4} v \leq 0$ in $H$ and that

$$
4^{-n b} n b \rho^{-1} \leq \frac{\partial v}{\partial x_{n}} \leq 4 n b \rho^{-1}
$$

at points $x=\left(a_{1}, a_{2}, \ldots, a_{n-1}, x_{n}\right)$ with $0 \leq x_{n} \leq \rho$. We note that $v>0$ on $B(a, \rho) \cap H$ and $v>1-2^{-n b}$ on $\partial B(a, \rho) \cap H$. From (3.3) and the maximum principle it follows that for $0 \leq x_{n} \leq \rho$,

$$
\begin{aligned}
\omega_{L_{4}}^{\left(a_{1}, a_{2}, \ldots, a_{n-1}, x_{n}\right)}(\Delta(0, \rho), D) & \leq\left(1-2^{-n b}\right)^{-1} v\left(\left(a_{1}, a_{2}, \ldots, a_{n-1}, x_{n}\right)\right) \\
& \leq 4 n b\left(1-2^{-n b}\right)^{-1} \frac{x_{n}}{\rho}
\end{aligned}
$$

thus $\omega_{L_{4}}^{x}(\Delta(0, \rho), D) \leq C(n, b) \frac{x_{n}}{\rho}$ on $T_{1}$. Because $u(x) \geq x_{n}(3 \rho)^{-b(n-1)-1}$ on $T_{1},(3.2)$ holds on $T_{1}$. It is easy to verify (3.2) on $T_{2}$.

Finally (3.1) follows from (3.2) and the maximum principle.

Remark 2. The kernel functions and the Green functions for a nondivergence operator can grow differently from their counterparts for divergence operators near the pole. We note from (3.1), (1.1), and the maximum principle that for $0<\rho<1$,

$$
c(n, b) \rho^{(n-1) b} \leq \omega_{L_{4}}^{(0, \ldots, 0,1)}(\Delta(0, \rho), H) \leq C(n, b) \rho^{(n-1) b},
$$

and that $(n-1) b$ may take any positive value. Moreover the exponent in $|x|^{-b(n-1)+1}$, the Green function at 0 when $b(n-1) \neq 1$, may take any nonzero value less than 1 ; in particular it may become positive for certain $b$ 's.

This unconventional behavior of the Green function has been used in [14], to construct large sets of vanishing capacity and small sets of positive capacity for certain operators (0.4); and it is this asymptotic behavior of $\omega_{L_{4}}(\Delta(0, \rho), H)$ that has motivated us to study the examples in this paper.

We shall follow the argument in $\S 2$; and put translates of $L_{4}$ on a sequence of hemispheres.

To prove Theorem 2 for nondivergence operators, let $S$ be a set on $\partial H$ in the class $\Pi\left(\left\{\alpha_{k}\right\},\left\{M_{k}\right\}\right)$ with

$$
M_{k} \geq 3+n K \gamma\left(n, K^{2}\right)^{-1} .
$$

As in $\S 2, l_{k}=\prod_{1}^{k} \alpha_{j}^{M_{j}}$; notations $\mathscr{C}_{k}, H_{k}, \mathbb{Z}_{k}, p(w), q(w), t(w), r(w), I(w)$, and $J(w)$ are also retained. 
Let $\left\{\varepsilon_{k}\right\}, 0<\varepsilon_{k} \ll \alpha_{k} l_{k-1}$, be a sequence of numbers to be explained later; and let $b(x)$ be a function continuous on $H, 1 \leq b(x) \leq K^{2}$, and

$$
b(x)= \begin{cases}K^{2}, & \text { on } \bigcup_{k} \bigcup_{w \in \mathbb{Z}_{k}}\left(p(w) \backslash B\left(w, \varepsilon_{k}\right)\right), \\ 1, & \text { on }\left(H \backslash \bigcup_{k} \bigcup_{w \in \mathbb{Z}_{k}} q(w)\right) \cup \bigcup_{k} \mathbb{Z}_{k} .\end{cases}
$$

Define, in $H$,

$$
\begin{aligned}
L_{5}(x) & =\left\{\begin{aligned}
b(x) \Delta-(b(x)-1) \sum_{i, j}\left(x_{i}-w_{i}\right)\left(x_{j}-w_{j}\right)|x-w|^{-2} \frac{\partial^{2}}{\partial x_{i} \partial x_{j}}, \\
\text { if } x \in q(w), \text { for some } w \in \mathbb{Z}_{k} \quad(k \geq 1)
\end{aligned}\right. \\
& \equiv \sum_{i, j} A_{i j}(x) \frac{\partial^{2}}{\partial x_{i} \partial x_{j}} .
\end{aligned}
$$

The introduction of $B\left(w, \varepsilon_{k}\right)$ in (3.4) is to guarantee the continuity of coefficients of $L_{5}$, which is a serious matter for operators of nondivergence form. Also the coefficients $\left(A_{i j}(x)\right)$ satisfy

$$
\left\|A_{i j}(x)-\delta_{i j}\right\|_{\infty} \leq b(x)-1 \leq K^{2}-1
$$

and the parameters of the ellipticity satisfy

$$
\Lambda(x) / \lambda(x)=b(x) \leq K^{2} .
$$

Following the argument in $\S 2$ and replacing (2.2) by (3.1), we obtain the analogue of $(2.10)$ for $L_{5}$ :

$$
\omega_{L_{5}}^{P}(J(w)) / \omega_{L_{5}}^{P}(I(w)) \leq C(n, K) \alpha_{k}^{(n-1) K} .
$$

Thus it follows from $(0.8)$ that the set $S$ has positive $\omega_{L_{5}}$-measure.

To prove Theorem 3 for nondivergence operators, we assume $(0.9)$ and, as before, that $\lim _{k \rightarrow \infty} \alpha_{k}^{K_{k}-1}=0$. Let $S \subseteq \partial H$ be a set in $\Pi\left(\left\{\alpha_{k}\right\},\left\{M_{k}\right\}\right)$ with $M_{k} \geq 3+2 n \gamma(n, 4)^{-1}$. Let $l_{k}=\prod_{1}^{k} \alpha_{j}^{M_{j}}$ and $0<\varepsilon_{k} \ll \alpha_{k} l_{k-1}$. Modify the definition of $b(x)$ so that

$$
b(x)= \begin{cases}K_{k}^{2} & \text { on } \bigcup_{w \in \mathbb{Z}_{k}}\left(p(w) \backslash B\left(w, \varepsilon_{k}\right)\right) \quad(k \geq 1), \\ 1 & \text { on } H \backslash\left(\bigcup_{k} \bigcup_{w \in \mathbb{Z}_{k}} q(w)\right) \cup \bigcup_{k} \mathbb{Z}_{k},\end{cases}
$$

$1 \leq b(x) \leq K_{k}^{2}$ on $\left\{x: l_{k} / 2 \leq x_{n} \leq l_{k-1} / 2\right\}$ and that $b(x)$ is continuous in $H$ (actually up to $\bar{H}$ ). Let $L_{6}$ be the operator formulated as in (3.5) for the new $b(x)$ instead. Then $\left(S, L_{6}\right)$ satisfies all the assertions in Theorem 3 provided that $\left\{\varepsilon_{k}\right\}$ are sufficiently small.

\section{Remarks on the Class II AND the PRoOF of Theorem 4}

In defining $\Pi\left(\left\{\alpha_{k}\right\},\left\{M_{k}\right\}\right)$, if a more restricted condition,

$$
l\left(I_{k, j}\right)=\prod_{1}^{k-1} \alpha_{j}^{M_{j}}
$$

is used instead of (0.7), then we obtain a subclass $\Pi^{\prime}$ such that every set in $\Pi^{\prime}$ contains a set of the form

$$
I_{1} \times I_{2} \times \cdots \times I_{j-1} \times E \times I_{j+1} \times \cdots \times I_{m}
$$


where $I_{k}$ 's are intervals and $E$ is a perfect set on $\mathbb{R} \quad(1 \leq j \leq m)$.

The more relaxed condition (0.7) allows the center of a cube in $\mathscr{C}_{k+1}$ to be shifted to any place in $S_{k}$ maintaining a distance $\frac{1}{2} \prod_{1}^{k} \alpha_{j}^{M_{j}}$ away from $\partial S_{k}$. There are sets $F$ in $\Pi\left(\left\{\alpha_{k}\right\},\left\{M_{k}\right\}\right)$ which meet at most countably many hyperplanes $\left\{x_{j}=a\right\} \quad(1 \leq j \leq m$ and $a \in \mathbb{R})$ at some relative interior points of $\left\{x_{j}=a\right\} \cap F$. Such examples will become apparent after a more complex example is built for Theorem 4 .

The fact that the center of a cube in $\mathscr{C}_{k+1}$ must maintain a certain distance from $\partial S_{k}$ puts restraints on the properties of sets in $\Pi\left(\left\{\alpha_{k}\right\},\left\{M_{k}\right\}\right)$.

In Theorem 4, we repeatedly shift the cubes and modify the boundaries $\partial S_{k}$. The sets so obtained are no longer in any $\Pi\left(\left\{\alpha_{k}\right\},\left\{M_{k}\right\}\right)$ class.

Proof of Theorem 4. For simplicity, we consider only $n=3$, i.e., $H=\mathbb{R}_{+}^{3}$ and $\partial H=\mathbb{R}^{2}$; and we only study the divergence operators with the assumption:

$$
\sum \alpha_{k}^{2}=\infty \quad \text { and } \quad \sum \alpha_{k}^{2 K}<\infty \text { for some } K>1 .
$$

Examples for the remaining cases can be built similarly.

Replacing $\alpha_{k}$ by a number at most twice its size we may assume that $\alpha_{k}^{-1}$ are odd integers.

For $x \in \mathbb{R}^{2}, a>0$, denote by $I(x, a)$ the closed square with vertices $x, x+(a, 0), x+(0, a)$, and $x+(a, a)$; denote by $\mathbb{Z}$ all lattice points on $\mathbb{R}^{2}$. Let $l_{0}=1, l_{k}=\prod_{1}^{k} \alpha_{j}^{M_{j}}$ with integers $M_{k}$ to be specified later, and let

$$
\mathscr{C}_{k}=\left\{I\left(l_{k-1} z, l_{k-1}\right): z \in \mathbb{Z}\right\} \quad(1 \leq k<\infty) .
$$

Note that each $I \in \mathscr{C}_{k}$ is composed of exactly $\alpha_{k}^{-2 M_{k}}$ squares from $\mathscr{C}_{k+1}$.

We call a line segment $x_{1}$-agreeable if it has length $l_{k}$ for some $k$ and its projection onto the $x_{1}$-axis contains an interval of the form $\left(j l_{k},(j+1) l_{k}\right)$ for some integer $j$. A square in $\mathbb{R}^{2}$ is called $x_{1}$-agreeable if two of its sides are $x_{1}$-agreeable segments. Define $x_{2}$-agreeable similarly. riors.

Two squares are called practically disjoint if they have mutally disjoint inte-

For an odd integer $k$, we define a horizontal shift $\mathscr{C}_{k}^{s}$ of $\mathscr{C}_{k}$ as follows:

$$
\mathscr{C}_{k}^{s}=\left\{I\left(l_{k-1}\left(i+j \alpha_{k}, j\right), l_{k-1}\right):(i, j) \in \mathbb{Z}\right\} ;
$$

and for an even $k$, we define $\mathscr{C}_{k}^{s}$ to be a vertical shift of $\mathscr{C}_{k}$ :

$$
\mathscr{C}_{k}^{s}=\left\{I\left(l_{k-1}\left(i, j+i \alpha_{k}\right), l_{k-1}\right):(i, j) \in \mathbb{Z}\right\} .
$$

Proposition 3. If $k$ is odd, any vertical line segment $\eta$ of length $\alpha_{k}^{-1} l_{k-1}$ intersects $\bigcup\left\{\alpha_{k} I: I \in \mathscr{C}_{k}^{s}\right\}$ on a set of length at least $\alpha_{k} l_{k-1}$; in the case that $\eta$ is the union of $\alpha_{k}^{-1}$ many $x_{2}$-agreeable segments, the intersection contains a segment of length $\alpha_{k} l_{k-1}$. If $k$ is even, any horizontal line segment $\xi$ of length $a_{k}^{-1} l_{k-1}$ intersects $\bigcup\left\{\alpha_{k} I: I \in \mathscr{C}_{k}^{s}\right\}$ on a set of length at least $a_{k} l_{k-1}$; in the case that $\xi$ is the union of $a_{k}^{-1}$ many $x_{1}$-agreeable segments, the intersection contains $a$ segment of length $\alpha_{k} l_{k-1}$.

The following properties can be obtained by induction. 
Proposition 4. (1) If $k$ is odd, squares in $\mathscr{C}_{k}^{s}$ are $x_{2}$-agreeable, and if $k$ is even, squares in $\mathscr{C}_{k}^{s}$ are $x_{1}$-agreeable.

(2) Each square in $\mathscr{C}_{k}^{s}$ is the union of exactly $\alpha_{k}^{-2 M_{k}}$ practically disjoint squares from $\mathscr{C}_{k+1}$; and each square in $\left\{\alpha_{k} I: I \in \mathscr{C}_{k}^{s}\right\}$ is composed of exactly $\alpha_{k}^{-2 M_{k}+2}$ practically disjoint squares from $\mathscr{C}_{k+1}$.

(3) For each $I \in \mathscr{C}_{k}^{s}$, there exist $I^{\prime}$ and $I^{\prime \prime} \in \mathscr{C}_{k+1}^{s}$ so that $I^{\prime}$ is not contained in $I, I^{\prime \prime}$ is not contained in $\alpha_{k} I$ but $I^{\prime} \cap I$ and $I^{\prime \prime} \cap \alpha_{k} I$ have nonempty interiors. Thus $I$ and $\alpha_{k} I$ are not entirely composed of squares from $\mathscr{C}_{k+1}^{s}$.

$$
\begin{gathered}
\text { Let } \mathscr{S}_{1}=\mathscr{C}_{1}^{s}, \mathscr{T}_{1}=\left\{\alpha_{1} I: I \in \mathscr{S}_{1}\right\}, S_{1}=\mathbb{R}^{2}, \text { and } T_{1}=\bigcup\left\{I \in \mathscr{T}_{1}\right\} ; \\
\mathscr{S}_{2}=\left\{I \in \mathscr{C}_{2}^{s}: I \text { does not meet } T_{1}\right\}, \\
\mathscr{T}_{2}=\left\{\alpha_{2} I: I \in \mathscr{S}_{2}\right\}, S_{2}=\bigcup\left\{I \in \mathscr{S}_{2}\right\}, T_{2}=\bigcup\left\{I \in \mathscr{T}_{2}\right\} ; \text { and } \\
\mathscr{S}_{k}=\left\{I \in \mathscr{C}_{k}^{s}: I \cap\left(T_{k-1} \bigcup\left(\mathbb{R}^{2} \backslash S_{k-1}\right)\right)=\varnothing\right\},
\end{gathered}
$$

$\mathscr{T}_{k}=\left\{\alpha_{k} I: I \in \mathscr{S}_{k}\right\}, S_{k}=\bigcup\left\{I \in \mathscr{S}_{k}\right\}$, and $T_{k}=\bigcup\left\{I \in \mathscr{T}_{k}\right\}$ for all $k \geq 3$. Let

$$
S=\bigcap_{1}^{\infty} S_{k}
$$

We claim that if

$$
\alpha_{k}^{M_{k}}<\frac{1}{2} \alpha_{k+1} \quad \text { for each } k \geq 1,
$$

then $S$ meets every horizontal line and every vertical line on a set of zero length.

Note from the construction that

$$
S_{k} \cap\left(\bigcup_{j=1}^{k-1}\left(\bigcup\left\{\alpha_{j} I: I \in \mathscr{C}_{j}^{s}\right\}\right)\right)=\varnothing .
$$

Let $\xi$ be any horizontal line, and consider any $x_{1}$-agreeable line segment $\eta$ on $\xi$ of length 1. It follows from the assumption (4.3) and Proposition 3 that $\eta \cap\left(\bigcup\left\{\alpha_{2} I: I \in \mathscr{C}_{2}^{s}\right\}\right)$ consists of at least $\alpha_{2} \alpha_{1}^{-M_{1}}-1$ many segments of length $\alpha_{2} \alpha_{1}^{M_{1}}$ each. They constitute a total length at least $\alpha_{2}^{2}-\alpha_{2} \alpha_{1}^{M_{1}}$. Note from (4.4) that $\eta \cap S_{3} \subseteq \eta \backslash\left(\bigcup\left\{\alpha_{2} I: I \in \mathscr{C}_{2}^{s}\right\}\right)$; thus

$$
l\left(\eta \cap S_{3}\right) \leq 1-\alpha_{2}^{2}+\alpha_{2} \alpha_{1}^{M_{1}} .
$$

Write $\eta \cap S_{3}$ as union of disjoint $x_{1}$-agreeable intervals of length $l_{2}=\alpha_{1}^{M_{1}} \alpha_{2}^{M_{2}}$ each. Call such an interval $\tau$. It follows from the assumption (4.3) and Proposition 3 again that $\tau \cap\left(\bigcup\left\{\alpha_{4} I: I \in \mathscr{C}_{4}^{s}\right\}\right)$ consists of at least $l(\tau) /\left(\alpha_{4}^{-1} l_{3}\right)-1$ many segments of length $\alpha_{4} l_{3}$ each. These intervals have a total length at least $\alpha_{4}^{2} l(\tau)-\alpha_{4} l_{3}=\left(\alpha_{4}^{2}-\alpha_{4} \alpha_{3}^{M_{3}}\right) l(\tau)$. Again, because of (4.4), $l\left(\tau \cap S_{5}\right) \leq$ $l(\tau)\left(1-\alpha_{4}^{2}+\alpha_{4} \alpha_{3}^{M_{3}}\right)$. Summing over all these $\tau$ 's and applying (4.3), we may conclude that

$$
\begin{aligned}
l\left(\eta \cap S_{5}\right) & =l\left(\eta \cap S_{3} \cap S_{5}\right) \leq l\left(\eta \cap S_{3}\right)-l\left(\eta \cap S_{3}\right)\left(\alpha_{4}^{2}-\alpha_{4} \alpha_{3}^{M_{3}}\right) \\
& \leq\left(1-\alpha_{2}^{2}+\alpha_{2} \alpha_{1}^{M_{1}}\right)\left(1-\alpha_{4}^{2}+\alpha_{4} \alpha_{3}^{M_{3}}\right) \leq\left(1-\frac{1}{2} \alpha_{2}^{2}\right)\left(1-\frac{1}{2} \alpha_{4}^{2}\right) .
\end{aligned}
$$


Continue by induction:

$$
l\left(\eta \cap S_{2 k+1}\right) \leq \prod_{1}^{k}\left(1-\frac{1}{2} \alpha_{2 j}^{2}\right) .
$$

Because $\left\{\alpha_{k}\right\}$ and $\left\{S_{k}\right\}$ are monotone decreasing, it follows from (4.1) that $l(\eta \cap S)=0$, and thus $l(\xi \cap S)=0$ for every horizontal line $\xi$.

Similarly $l(\xi \cap S)=0$ for every vertical line $\xi$. This verifies the claim.

Let $b=1+4(n-1)\left(K^{4}-1\right), \gamma=\gamma(3, b)$ be the constant chosen in Lemma 4 , and assume that

$$
M_{k} \geq 3+n K \gamma^{-1}, \quad k \geq 1 .
$$

Following step by step the construction of $L_{2}$ in $\S 2$, we build a divergence operator $L_{7}$ in $H$, associated with the current selections of cubes $\mathscr{C}_{k}^{s}$, hyperplanes $x_{3}=l_{k}$, elevated centers $\mathbb{Z}_{k}=\left\{\left(y, l_{k}\right): y\right.$ center of a cube in $\left.\mathscr{C}_{k}^{s}\right\}$, and hemispheres $p(w)$ of center $w \in \mathbb{Z}_{k}$ and radius $l_{k-1} / 4$. Recall that the ratio $\Lambda / \lambda$ of the ellipticity parameters depends on $K$ and $n=3$ only.

Recall also that $P=(0,0,1)$, and denote $\omega_{L_{7}}^{P}$ by $\omega$ for simplicity. Following the argument for (2.10), we deduce that

$$
\omega\left(\alpha_{k} I\right) / \omega(I) \leq C_{1}(K) \alpha_{k}^{2 K}, \quad I \in \mathscr{C}_{k}^{s},
$$

for all $k>k_{0}$. Also it follows from the doubling property of $\omega$ that there exists a number $\theta, 0<\theta<1$, depending only on $K$ so that

$$
\omega\left(\frac{1}{2} I\right) \leq \theta \omega(I)
$$

for any square $I \subseteq \partial H$. Let $\theta_{k}=\theta$ for $k \leq k_{0}$ and

$$
\theta_{k}=\min \left\{\theta, C_{1}(K) \alpha_{k}^{2 K}\right\}<1, \quad \text { for } k>k_{0},
$$

we have

$$
\omega\left(\alpha_{k} I\right) / \omega(I) \leq \theta_{k} .
$$

Note from the assumption (4.1) that $\sum \theta_{k}<\infty$, and let

$$
\varepsilon=\prod_{1}^{\infty}\left(1-\theta_{k}\right)
$$

It follows from the doubling property of $\omega$ again that there exists a number $\delta=\delta(K, \varepsilon)<\frac{1}{100}$, so that

$$
\omega((1+\delta) I) \leq(1+\varepsilon) \omega(I)
$$

for any square $I \subseteq \partial H$.

Assume, in addition, that

$$
\sum_{k+1}^{\infty} \alpha_{j}^{M_{j}}<\delta n^{-1} \min \left\{\alpha_{k}^{M_{k}}, \alpha_{k+1}\right\} \quad \text { for each } k \geq 1 ;
$$

and note that (4.8) is a very weak assumption because $\left\{\alpha_{k}\right\}$ is decreasing.

Before we proceed to prove $\omega(S)>0$, we need to introduce more notations. For each $k$, and $j \geq k$, let $T_{k, k}=T_{k}$,

$$
T_{k, j+1}=\bigcup\left\{I \in \mathscr{C}_{j+1}^{s}: I \cap T_{k, j} \neq \varnothing\right\},
$$


and note that $T_{k, j+1} \supseteq T_{k, j}$. Let $T_{k, j+1}^{\prime}=T_{k, j+1} \backslash T_{k, j}$, and $\widetilde{T}_{k}=\bigcup_{j=k}^{\infty} T_{k, j}$. In view of (4.7),

$$
\widetilde{T}_{k} \subseteq \bigcup\left\{(1+\delta) I: I \in \mathscr{T}_{k}\right\} \stackrel{\text { call }}{=} \widetilde{T}_{k} ;
$$

from (4.8), (4.9), and the definitions of $T_{k}$ and $T_{k, j+1}$, it follows that $\left\{\widetilde{T}_{k}\right\}$ are mutually disjoint. Observe also that

$$
\begin{gathered}
S_{k+1}=S_{k} \backslash \bigcup_{i=1}^{k} T_{i, k+1}=S_{k} \backslash \bigcup_{i=1}^{k} T_{i, k+1}^{\prime}, \\
\mathbb{R}^{2} \backslash S_{k} \subseteq \bigcup_{1}^{k-1} \widetilde{T}_{j},
\end{gathered}
$$

and

$$
T_{k} \subseteq S_{k} \subseteq \mathbb{R}^{2} \backslash \bigcup_{1}^{k-1} T_{j}
$$

Note from (4.6) and (4.11) that

$$
\omega\left(T_{k}\right) \leq \theta_{k} \omega\left(S_{k}\right) \leq \theta_{k}\left(1-\sum_{1}^{k-1} \omega\left(T_{j}\right)\right) .
$$

Consequently, $\omega\left(T_{1}\right) \leq \theta_{1}$,

$$
\omega\left(T_{1}\right)+\omega\left(T_{2}\right) \leq \omega\left(T_{1}\right)+\theta_{2}\left(1-\omega\left(T_{1}\right)\right) \leq \theta_{1}+\theta_{2}\left(1-\theta_{1}\right),
$$

because $\theta_{2}<1$. Assume that $\sum_{1}^{k-1} \omega\left(T_{j}\right) \leq 1-\prod_{1}^{k-1}\left(1-\theta_{j}\right)$; then

$$
\sum_{1}^{k} \omega\left(T_{j}\right)=\sum_{1}^{k-1} \omega\left(T_{j}\right)+\theta_{k}\left(1-\sum_{1}^{k-1} \omega\left(T_{j}\right)\right) \leq 1-\prod_{1}^{k}\left(1-\theta_{j}\right)
$$

because $\theta_{k}<1$.

Because of (4.7) and (4.9), $\omega\left(\widetilde{T}_{k}\right) \leq(1+\varepsilon) \omega\left(T_{k}\right)$. Therefore, it follows from (4.10) and (4.12) that

$$
\omega\left(\mathbb{R}^{2} \backslash S_{k}\right) \leq \sum_{1}^{k-1} \omega\left(\widetilde{T}_{j}\right) \leq(1+\varepsilon)\left(1-\prod_{1}^{k-1}\left(1-\theta_{j}\right)\right) ;
$$

thus

$$
\begin{aligned}
\omega\left(S_{k}\right) & \geq 1-(1+\varepsilon)\left(1-\prod_{1}^{k-1}\left(1-\theta_{j}\right)\right)=(1+\varepsilon) \prod_{1}^{k-1}\left(1-\theta_{j}\right)-\varepsilon \\
& >(1+\varepsilon) \prod_{1}^{\infty}\left(1-\theta_{j}\right)-\varepsilon=\varepsilon^{2}>0 .
\end{aligned}
$$

From this it follows that $\omega(S)>0$.

Clearly, $S$ is $\left\{\alpha_{k}\right\}$-porous.

The set $S$ constructed in (4.2) has all the properties required in Theorem 4, provided that $M_{k}$ 's are chosen to satisfy (4.3), (4.5), and (4.8).

In closing, we mention a question of R. Kaufman. Does this set $S$ meet every Lipschitz arc on a set of zero length? If the answer is no, could one construct a set which has this property in addition to all those in Theorem 4 ? 


\section{REFERENCES}

1. T. Barceló, A completely singular harmonic measure for a nondivergence form elliptic operator with a drift, Comm. Partial Differential Equations 14 (1989), 931-958.

2. $\ldots$, On the harmonic measure for nondivergence elliptic equations, Comm. Partial Differential Equations 16 (1991), 1367-1422.

3. P. Bauman, Positive solutions of elliptic equations in nondivergence form and their adjoints, Ark. Mat. 22 (1984), 153-173.

4. A. Beurling and L. Ahlfors, The boundary correspondence under quasiconformal mappings, Acta Math. 96 (1956), 125-142.

5. L. Carleson, The extension problem for quasiconformal mappings, Contributions to Analysis, Academic Press, New York, 1974, pp. 39-47.

6. L. Caffarelli, E. Fabes, and C. Kenig, Completely singular elliptic-harmonic measures, Indiana Univ. Math. J. 30 (1981), 917-924.

7. L. Caffarelli, E. Fabes, S. Mortola, and S. Salsa, Boundary behavior of nonnegative solutions of elliptic operators in divergence form, Indiana Univ. Math. J. 30 (1981), 621-640.

8. E. Fabes, N. Garofalo, S. Marin-Malave, and S. Salsa, Potential theory and Fatou theorems for some nonlinear elliptic equations, Rev. Mat. Iberoamericana 4 (1988), 227-252.

9. D. Gilbarg and N. S. Trudinger, Elliptic partial differential equations of second order, 2nd ed., Springer-Verlag, Berlin, 1983.

10. W. Littman, G. Stampacchia, and H. F. Weinberger, Regular points for elliptic equations with discontinuous coefficients, Ann. Scuola Norm. Sup. Pisa 17 (1963), 43-77.

11. L. Modica and S. Mortola, Construction of a singular elliptic-harmonic measure, Manuscripta Math. 33 (1980), 81-98.

12. L. Modica, S. Mortola, and S. Salsa, A nonvariational second order elliptic operator with singular elliptic measure, Proc. Amer. Math. Soc. 84 (1982), 225-230.

13. P. Tukia and J. Väisälä, Quasiconformal extension from dimension $n$ to $n+1$, Ann. of Math. (2) 115 (1982), 331-348.

14. J.-M. Wu, Examples of capacity for some elliptic operators, Trans. Amer. Math. Soc. 333 (1992), 379-395.

15. $\ldots$, Null sets for doubling and dyadic doubling measures, Ann. Acad. Sci. Fenn. 18 (1993),

Department of Mathematics, University of Illinois, 1409 West Green Street, Urbana, ILLINOIS 16801 